\title{
La letteratura migrante come diario
}

\author{
Alma Hafisi \\ Università di Scutari, Facoltà di Lingue Straniere
}

Članek nadaljuje moje prvo raziskovanje migrantske literature albanskih izseljencev v Italiji, ki je dokazovalo ozmozo med spominom, identiteto in spreminjanjem. V njem pa se osredotočam na temo pogleda na tej in oni strani meje z analizo dnevniških romanov albanskih izseljencev v Italijo. Izseljenci trpijo zaradi prehajanja meje. Moledujejo za mejo, ki pa jih zavrača. Zapustijo svojo domovino v zameno za drugo, boljšo, kjer pa se počutijo zavrnjeni, nesprejeti, nepovabljeni. Gre za dolgo potovanje metamorfoze gledišča, iz katerega so opazovani, iz rojstnega kraja proti novi domovini. Va e non torna (Pojdi in se ne vrni) in Senza bagagli (Brezkovčkov) sta dva dnevniška romana, neke vrste stik med lastno fizično eksistenco in spominom, zrcalo, ki pomaga izseljencu živeti dinamiko spreminjanja z veliko mero iskrenosti in poštenosti, in po možnosti modrosti.

Ključne besede: migranti, meja, literatura, dnevnik, opazovanje.

Following my first reflection on the migratory literature of Albanian writers who have written their works in Italian language, such as the osmosis of memory, identity and transformation, my short paper is focused on the theme of looking, beyond and across the frontiers, through the diary novels of Albanian emigrants in Italy. Migrants suffer from frontiers syndrome. They look at them imploringly and respond to them aversively. They leave their homeland for something new which would be better, and instead they find themselves rejected, unwanted, uninvited. It is a journey along the metamorphosis of the way of being observed from the country of origin to that of arrival. Goes and does not come back, Without luggage, these are the novels that should be read as an emigrant's diary, as a link between one's physical existence and memory, a mirror that helps the emigrant to live the dynamics of transformation with the utmost sincerity and honesty, and possibly wisely.

Key words: emigrant, frontiers, literature, diary, observation

$\mathrm{V}$ arcare le frontiere di uno stato totalitario come era l'Albania, prima degli anni Novanta, era visto come un miracolo o un peccato mortale. Pochi, i più fortunati, ci sono riusciti. Agli altri era permesso solo supporre quello che esisteva al di là del confine, oppure, indottrinati dalla propaganda del regime, erano costretti a cancellare l'idea che il mondo continuava a esistere oltre il muro. Era l'unico modo di sopravvivenza psicologica e fisica. Partendo da queste premesse, il mondo oltre la frontiera non era solo l'allungamento dello stesso pianeta nel tempo e nello spazio, ma più passavano gli anni che portavano l'Albania nell' isolamento totale, più diventava un altro pianeta, il paradiso per alcuni, l'inferno per gli altri, comunque qualcos'altro.

La caduta del muro di Berlino ha avverato il sogno degli albanesi e, poco dopo, il I991, è stato l'anno della speranza, della liberazione 
che vide centinaia di migliaia di profughi sbarcare sulle coste italiane dell'Adriatico. Cronache di giornali, testimonianze, impressioni e racconti di politici, di addetti ai centri di accoglienza si permearono dappertutto, ma quella che ha restituito tutta la sofferenza degli albanesi che attraversarono il 'mare confine', è stata la letteratura scritta in italiano dagli stessi albanesi. Scrittori di nuova generazione, emigrati in Italia, allargano la geografia della letteratura transnazionale di tradizione oramai in Italia. Nomi come Ron Kubati, Elvira Dones, Anilda Ibrahimi, Gëzim Hajdari, Ornela Vorpsi, Artur Spanjoli, Leonard Guaci raccontano storie vissute personalmente o appartenenti alla memoria collettiva, risalenti al tempo del regime e del tramonto della dittatura, ma anche agli anni dell'emigrazione, che oltre ad essere lettura di intrattenimento sono anche testi degni di essere studiati per la loro natura psicologica. Della vasta produzione narrativa degli autori sopra elencati prendiamo in esame due romanzi: Va e non torna (Kubati 2004) di Ron Kubati e Dashuri e huaj (Dones 2015) ${ }^{2}$ di Elvira Dones, tradotto anche in italiano con il titolo Senza bagagli, entrambi romanzi di esordio.

Tenendo conto di quanto diceva Armando Gnisci, "l'esodo incide profondamente la vita di un uomo, tanto da dividere la sua esistenza in ante-migrazione ed in post-migrazione" (Gnisci 2003,8 ), è ovvio che i migranti che scrivono non possano fare a meno di collegarsi continuamente a tale situazione di sospensione. I due romanzi citati, di taglio fortemente autobiografico, si riferiscono al periodo albanese, coincidente con la dittatura di Enver Hoxha, e si concludono con

\footnotetext{
Ron Kubati è nato a Tirana nel 197 I in una nota famiglia di dissidenti. Arrivato in Italia nel I99I, vive in provincia di Bari dove continua a studiare (dottorato di ricerca in filosofia) e si dedica alla scrittura. Lavora come traduttore. In Italia ha pubblicato "Venti di libertà e gemiti di dolore" (1991), in Albania la raccolta di poesie "Tra speranza e sogno" (1992). Collabora con diversi quotidiani (La Gazzetta del Mezzogiorno e la Repubblica di Bari, inserto pugliese de La Repubblica); è membro della giuria letteraria del Premio Balcanica che viene assegnato a rotazione in diverse capitali dei Balcani.

2 Elvira Dones è nata a Durrazzo e cresciuta a Tirana (Albania). All'età di dieci anni scrive un libro (di una dozzina di pagine) che intitola "Romanzo". A sedici anni inizia a condurre programmi televisivi. Nel 1988 lascia il suo paese - a quel tempo ancora una dittatura stalinista - e si stabilisce in Svizzera. Nel 1997 pubblica il suo primo vero romanzo Dashuri e huaj (Senza bagagli).
}

l'abbandono e il ritrovarsi nella terra sull'altra sponda del mare.

Kubati si impegna a disegnare la propria vita a partire da alcuni semplici ricordi dell'infanzia ai tempi della dittatura, quando ancora non ne aveva preso coscienza, segnata da un evento terribile, l'arresto e l'incarceramento del padre, ex funzionario dello stato, per proseguire con l'adolescenza e la visione della TV proibita, le feste con balli organizzate di nascosto, l'imposizione ufficiale degli studi da seguire, senza possibilità di scelta, e finire la narrazione con la vita studentesca in Italia tra lavori, studi e sforzi di sopravvivenza, intercalando in un andirivieni tra presente e passato vicende personali e pubbliche in Albania, l'ultima delle quali è la fuga dal paese.

Elvira Dones, la cui biografia somiglia moltissimo a quella della protagonista del suo romanzo, un'operatrice televisiva, ci regala una doppia storia; la storia dell'Albania tra gli anni Ottanta e Novanta, nel periodo di passaggio dal regime oppressivo a quello di una apparente democrazia, con la storia di un popolo maltrattato passata inosservata a tanti paesi vicini, intrecciata a una storia d'amore, di un amore a prima vista, di un forte sentimento, di quelli che cambiano la vita. Nella sua storia è plasmata quella di tanti uomini e donne che hanno solcato l'Adriatico fiduciosi in un futuro migliore, pieni di speranza e che hanno dovuto affrontare le mille difficoltà di essere profughi, sradicati, ripudiati in patria, disprezzati dalle persone con le quali per anni hanno condiviso la loro vita.

I due romanzi possono essere narrati e riassunti su tre livelli cardine che a prima vista sembrano abbastanza semplici, ma che sullo sfondo nascondono un mondo affascinante che si stratifica anche nella realtà che circonda il lettore per il tempo in cui sono oggetto di lettura: quello temporale, scandito a sua volta in tre parti, il periodo del soggiorno in Albania sotto la dittatura comunista, l'esodo, il processo di integrazione nella nuova realtà acquisita; quello spaziale, la patria in relazione alla dittatura, che è un simbolo di prigione collettiva e della presa di coscienza, la costruzione della mobilità all'estero, cioè lo 
spazio vuoto del viaggio verso qualche altra dimora, infine il paese d'arrivo; quello psicologico, realizzato attraverso la prospettiva, l'esperienza e naturalmente l'azione dei protagonisti, Elton e Klea Borova.

Appare evidente l'osmosi fra l'elemento temporale e spaziale, tanto da avvalersi di una nuova 'nozione topologica', secondo la studiosa Anna Federici, cronologica e puntuale insieme, "filo che si dipana dal presente alla proiezione utopica, ma è anche punto fermo per ogni momento di un'esperienza iniziatica oramai acquisita" (Federici 20II).

\section{La sindrome delle frontiere e l'abbandono}

Con la caduta della dittatura gli albanesi si sono trovati senza punti di riferimento politici e culturali, e molti sono rimasti disorientati, osserva la studiosa Maria Cristina Mauceri (Mauceri 2013, I85-200). Questa situazione di disordine e di disorientamento inizia con l'abbandono del Paese.

Il porto è circondato sia dalla polizia sia dai soldati. Non conosco l'entrata del porto. Mi yengono in aiuto i soldati. Lasciamo a loro orologi, soldi e qualsiasi oggetto loro vogliano..././Dopo alcuni minuti, siamo nelle vicinanze di una grande nave... /./ che si chiama Legend, ... /./Dichiariamo occupata la nave. ... /. La nave era enorme e occuparla non era stato poi così difficile. In fondo le cose basta volerle. Conil passare del tempo la nave fu affrancata anche dall'altro lato e gli occupanti aumentarono... /./ qualche ora dopo i visi conosciuti aumentarono e pian piano cominciarono gli abbracci. 'Anche tu?... /./ guarda chi c'è.' A questo punto il genere di domanda cominciò a cambiare: 'Hai visto mio fratello? Hai visto la mia ragazza? ... /./. Il mattino dopo, quando ci siamo tutti e siamo in migliaia, la nave si muove. Il mare la trascina verso non si sa dove. Non siamo noi ad attraversare il mare. È il mare che si fa attraversare. /./ Già in partenza, siamo digiuni più di ventiquattro ore. Non capiamo più niente (Kubati 2004, 191-193).
Sono queste le pagine che fanno rivivere la fuga verso l'Italia, desiderata da lungo tempo e programmata mille volte, la finalizzazione di un lungo tentativo che il protagonista del romanzo Elton, fa assieme a molti suoi concittadini per sferrare l'ultimo colpo al crollo del regime. Studente nell'Albania di allora, l'autore protagonista Ron Kubati realizza un filmato documentario che comprende tutti gli episodi problematici che precedettero i grandi cambiamenti. Nella sua ottica di ventenne, più che la caduta del muro di Berlino era stata la spettacolare caduta di Ceaușescu a stimolare la fantasia degli albanesi, che egli fa sventolare davanti agli occhi del lettore in ordine cronologico. La prima a farne le spese fu la statua di Stalin; La voce del popolo, il giornale del partito del lavoro, concesse la pubblicazione di articoli polemici come mai prima, la gente seguiva sempre più apertamente $L a$ voce dell'America nella sua edizione in lingua albanese, la zona residenziale delle ambasciate, attaccate una all'altra, divenne improvvisamente luogo di passaggio e di attrazione fino a quando il cordone della polizia che faceva la guardia fu rotto ed i più agitati oltrepassarono $i$ cancelli di quella cubana e rumena, ma le preferite furono quella italiana, tedesca e francese, da dove le persone venivano trasportate al porto di Durazzo per l'imbarco verso l'Europa. La città degli studenti nella periferia di Tirana, diventato luogo di pellegrinaggio per insoddisfatti e no, ospitava di giorno in giorno minatori, operai, intellettuali, gente semplice. L'ultimo presidente comunista aveva accolto la richiesta del pluralismo politico sotto la pressione delle proteste popolari che avevano come centro magnetico lo sciopero degli studenti. Il grande crollo è descritto metaforicamente dal protagonista, testimone oculare di quasi tutte le proteste, con gli slow motion che caratterizzano la caduta rovinosa della statua del dittatore: "Enver Hoxha puntò i piedi per un po', poi divenne insicuro, traballante, infine cadde sciaguratamente senza schiacciare nessuno. Piazza Scanderbeg urlò come mai prima. Tutti desideravano vedere Enver Hoxha da un'altra angolatura: steso per terra” (Kubati 2004, I7I). 
Per Dones abbandonare il paese è stata un'avventura diversa. Vive la dittatura da una diversa posizione sociale e viene trascinata nell'avventura della grande fuga da un diverso punto di collocazione geografica rispetto a Kubati. La sua è la storia di Klea Borova, operatrice della televisione di Stato, di alto profilo pubblico, madre, non adeguata però alle direttive socialiste del socialismo in quanto divorziata, donna dall'aspetto e con vestiti appariscenti nell'Albania anni Ottanta di Enver Hoxha. Durante un privilegiato e brevissimo viaggio di lavoro scopre il mondo lontano dall'oppressione, illuminato da un raggio improvviso di luce, quale è la Danimarca, che la incanta con la varietà e l'abbondanza dei cibi, con gente vera e priva di complessi, con la libertà di scegliere i libri, la libertà di espressione, soprattutto, con i riccioli del giornalista italo-svizzero Yves Montalban. Diventa lui motivo di vita e di professione, destinatario di una corrispondenza formale (finge di scrivergli cartoline), dedicatario di un diario in inglese nascosto sotto il materasso per tenerlo all'insaputa dei genitori, perfino del figlio affinché non possa pensarne l'esistenza.

Una seconda inaspettata occasione in cui le viene proposto di andare all'estero, nell'Italia di fronte questa volta, a Milano, introduce il momento giusto per telefonare a Ives che raggiunge in un paio di ore, un suo sguardo che vale mille parole basta per salire in macchina e rifugiarsi nella Svizzera italiana, senza farsene prima un'idea, senza bagagli, provocando il suo dramma, l'abbandono del piccolo figlio Anthony e della famiglia in Albania. Lei che si era opposta quotidianamente al regime con le sue maniere e attitudini, ora segue da lontano la caduta del regime, aspetta impaziente le notizie diffuse dalle agenzie di stampa dell'Occidente. Le Monde, La Repubblica, tutti i quotidiani europei si erano accorti fortuitamente dell'Albania. Secondo questi centinaia di albanesi avevano fatto irruzione nelle ambasciate straniere a Tirana, quelle dei paesi dell'Occidente ma anche quelle dei paesi dell'Est. Quelle centinaia erano in realtà cinquemila persone che il governo albanese, tramite una pletora di comunicati contraddittori trasmessi da Radio Tirana, presentava come "malviventi e teppisti" e definiva la loro evasione in massa come un gesto di disturbo e mirato a confondere la situazione in Albania. La sera dell'irruzione degli albanesi nelle ambasciate lei si trovava a Locarno, al concerto di Andreas Vollenweider, che seguiva con occhi sprofondati nella "penombra delle strade di Tirana", afflitta dal panico che si trasformava in lacrime e parole disconnesse.

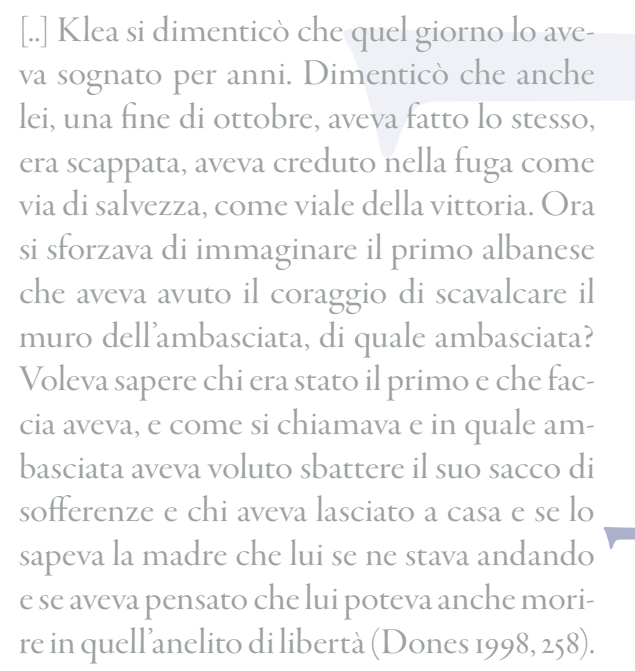

\section{La nevrosi del successo}

Il modo nel quale l'emigrante abbandona la terra nativa gli dà qualcosa dello splendore di un eroe. Così si sentivano i connazionali di Kubati quando sbarcavano sulle coste italiane nel 1991, perché avevano sfidato il regime, ed a loro parere l'Occidente li avrebbe dovuti risarcire delle sofferenze subite. L'emigrante si sente una creatura fragile nel suo vivere, confusa ed a volte anche ridicola. Nella sua psiche succedono molte cose. Paura, riluttanza, violenza della fuga e del primo contatto con un paese sconosciuto, ira, nostalgia della patria e, allo stesso tempo, sua negazione, sensi di colpa e rabbia risiedono nell'anima dell'emigrante. La reazione più istintiva è il silenzio. L'emigrante è un essere complicato, si circonda di insicurezza, per cui non cede alla narrazione. Si sente esposto al rifiuto, all'indifferenza, non protetto, sfruttato. Ragiona tra sé e si con- 
vince che la sua testimonianza non vale per nessuno (Kapllani 2016, 86).

Una reazione alternativa al silenzio è il rischio, mostrarsi e introdursi, narrare il proprio viaggio doloroso e avventuroso. Potrebbe essere più pericoloso, ma di sicuro vale più del silenzio. Le aspettative non sono grandi, si pretende solo di essere capiti. E per capire un emigrante va raccontata la sua storia precedente, dell"anti-emigrazione', gli antefatti dell'esodo. L'emigrante sente il bisogno di parlare, di sfogarsi anche con sé stesso, di confessarsi, e il diario ne costituisce una forma. Non a caso prendono via una produzione di romanzi, diari di scrittori transnazionali. "Nessuno avrebbe letto le sue cretinate" pensava Klea seduta davanti al computer che aveva solo dischi e memorie mute che, volendo, cancellava in un attimo, cosa che non poteva fare con il passato, con la sensazione di non valere in quella terra. "Avrebbe provato. Non sapeva ancora se avesse cose da raccontare alla gente, agli altri, al mondo là fuori. Ma aveva cose da dire a sé stessa. E alla terra da cui proveniva. E quello era il modo migliore, il più desiderabile, il più personale, per dire quanto serbava in sé. [...] Cosa sto facendo? [...] Scrivo, pensò nella lingua che non le apparteneva e che spesso, involontariamente, violava la sua. Shkruaj" (Dones 1998, 280).

Elton, un ragazzino, non appena si era reso conto dei primi amori, per Keti "una bambina veramente bellina” e contemporaneamente per la maestra dell'asilo che "portava un profumo molto piacevole”, dimenticata Elona, compagna delle elementari, per Eltona, la nuova arrivata in classe, dai "jeans blu”, "lo zaino e i pennarelli colorati" che nessuno aveva, scrive del primo momento dello scontro con la dittatura. Suo padre viene incarcerato insieme ai due cugini, funzionari dello Stato, e lui viene esiliato a Durazzo dalla nonna materna, strappato alla famiglia per il suo bene, per evitare gli insulti dei bambini del vicinato e la battaglia che Elton avrebbe dato loro.

Bastava ciò per esporlo allo sguardo di tutti e per farlo sentire diverso; un bambino che viene escluso da tutta la classe, invitata a casa di uno che compiva gli anni ${ }^{3}$, un ragazzino aspirante alla carriera di calciatore che non viene preso, certamente non per la sua mancata bravura, figlio di due genitori divorziati ${ }^{4}$, controllati fino nella privacy. "L'entrata di una prigione era un grosso portone e l'uscita era una cruna d'ago" (Kubati 2004, 49). È questo detto filosofico di sua madre a guidarlo nella vita adolescenziale. Tante eccezioni, tante carenze non solo economiche. Non avrebbe nemmeno immaginato che per la moglie di un condannato era meglio non "farsi notare", non tenere i capelli biondi, "meglio oscurarli”, che era proibito avere una moglie ungherese, tanto peggio russa, altrimenti erano guai, come accaduto ad alcuni detenuti come suo padre. Le visite in carcere, la descrizione della sofferenza fisica e morale lì dentro, sono un'esperienza drammatica a parte. Ciò che non ti uccide ti rende più forte. Di qui, nella solidarietà con Edmond, figlio di Ilir incarcerato, con Iris, anche lei figlia di un dissidente, con figli di altri detenuti, scaturisce la forza per portare avanti quella esistenza, stranamente chiamata vita, tra i ricordi che hanno in comune ed i sogni che fanno insieme per fuggire verso l'Occidente.

Il regime imponeva l'egualitarismo a cui non poteva sottrarsi nemmeno l'altra categoria della popolazione, i non dissidenti, i privilegiati, rappresentata da Klea Borova, l'alter ego della Dones (Mauceri 2009, 85-107) ${ }^{6}$. Suo padre aderi-

\footnotetext{
3 Escluso perché non aveva denunciato il suo amico Luke sentendolo dire che "le gomme da masticare, trovabili solo all'estero, sarebbero state prodotte anche in Albania, una volta morto zio Enver".

$4 \quad$ Il divorzio finto della coppia era praticabile durante la dittatura per il bene dei figli e per risparmiare al resto della famiglia la persecuzione. I genitori di Elton divorziano formalmente dopo la condanna del padre a dieci anni di galera, una tristezza per Elton non sapendo come stavano esattamente le cose, un sollievo per suo zio, che temeva il peggio.

5 La casa veniva visitata ogni tanto da gente non invitata, tanto meno desiderata, che vi entrava durante la loro assenza per installare o cambiare le microspie.

6 "Senza bagagli presenta diversi aspetti che rivelano la sua affinità con le narrazioni autobiografiche. Ė un testo che ha permesso a Dones di elaborare il lutto dell'abbandono dell'Albania. Si è anche notato come la narrazione talvolta assuma il tono di una confessione attraverso la quale la scrittrice vuole liberarsi dal senso di colpa per aver lasciato il figlio. Inoltre accomuna questo romanzo a un testo autobiografico la testimonianza e la riflessione sulla condizione diasporica, una condizione che la scrittrice al momento della stesura del libro iniziava a condividere con altri connazionali. [...] Senza bagagli è
} 
sce al Partito, lei lavora nel centro cinematografico, per cui gode del privilegio di viaggiare, di una buona reputazione nella società e della possibilità di maggiore guadagno rispetto alle altre professioni. Il tutto pesa poco rispetto alle difficoltà della dittatura. Lei è anticonformista e lo manifesta nel suo modo di vestire, vestiti attillati, minigonna, colori accesi, bavero rialzato, che provocano alcune riunioni dell'organizzazione del Partito, gode della libertà di gestione della propria vita e può decidere autonomamente di divorziare dal marito, un noto medico, senza un motivo sostanziale, gode inoltre di un impegno professionale privo di ideologie. La mancanza di rispetto per le norme morali socialiste pone Klea allo sguardo di tutti. In ambito professionale è sorvegliata sul piano politico da qualche membro del Partito, dalla polizia segreta, Sigurimi. Suscita la curiosità dei vicini in quanto donna sola, affascinante, di cui sorvegliano la vita privata. Aveva "pregato decine di volte di non arrabbiarsi con la curiosità malata di Tirana ", ma senza risultato perché sarebbe stato come privare la gente del cibo e dell'aria.

Forse lo sguardo in Dones, come nota la studiosa Cristina Maria Mauceri, non è solo una pura curiosità, ma anche un modo per scoprire altri modelli di vita. L'atmosfera che precede il primo viaggio di Klea all'estero è piena di raccomandazioni delle amiche di "guardare (la Danimarca) anche per loro" oppure di richieste di portare gomma da masticare per i bambini. Il clima sociale e politico soffocante a Tirana ed i

una riscoperta delle proprie radici e nello stesso tempo un sofferto requiem per un'identità perduta che solo attraverso la scrittura può essere ricostruita".

7 "L'interesse e i pettegolezzi inarrestabili della gente avevano iniziato a soffocarla. Questo fa l'amore con quella, quell'altro ha avuto uno zio in carcere sei mesi per furto, quella si prende per i capelli con la suocera, e quella poi, che si concia come una donna di strada e il marito che sta a guardare, quella Klea della televisione, all'ultimo concerto avete visto cosa s'era messa addosso? Avrebbe fatto meglio a metterselo in testa il vestito, è impossibile che sia una così brava ragazza come finge di essere, sembra un angelo ma chissà cosa nasconde, che a noi non la dà a bere con quelle furbizie, chissà dove lo tiene l'amante, è impossibile che non se la spassi con tutto quel teatro e quella televisione. Eh figliola, eri bellissima ieri sera in televisione, che tivenga un colpo, sembravi una stella. E poi, anche così sei splendida, beato chi ti avrà. Tutto questo le era diventato insopportabile." (Dones 1998, 70). rammarichi di Klea, come il suo rapporto con l'ex marito Giorgio, la tristezza inguaribile del figlio Anthony, la tragedia della sua amica Linda ${ }^{8}$, che non vuole «morire dentro», la carne bovina introvabile, l'amore per Yves lontano, sono ragioni più che convincenti per la decisione tanto pensata e improvvisamente presa di abbandonare l'Albania, il cui cadavere verrà descritto dopo, su un foglio bianco, per l'ufficio generale dei profughi, mentre spiegava il motivo della sua fuga:

"Le toccava scrivere la lettera - rendiconto - confessione più difficile della sua vita. [..] scrisse di donne costrette a divorziare dai mariti, accusati di tradimento della patria, per salvare i figli dai campi di lavoro. Scrisse di qualcuno che era stato sbattuto in prigione a quattordici anni, era il 1950, per una poesia d'amore inviata alla compagna di banco. Era ancora in carcere, condannato per 'amore borghese'. Il prigioniero non aveva mai toccato quella ragazza nemmeno con un dito. Scrisse di famiglie distrutte dal marito che aveva denunciato la moglie e della moglie che aveva denunciato i figli per "agitazione e propaganda contro il Partito", scrisse della fame dei bambini e di libri proibiti, del controllo sistematico delle antenne tivù e dei temi obbligatori a scuola: 'In Albania i bimbi sono i fiori della vita'. Il cadavere dell'Albania giaceva sul foglio bianco" (Dones 1998, 225).

\section{Amore e odio della Patria}

Il contatto con l'Occidente ha finalmente avverato il sogno di tanti albanesi. Lunga l'attesa, grande il sacrificio, pesante la vita sotto la dittatura, altrettanto profonda la delusione. Non basta trovarsi dall'altra parte per trovare la libertà. Klea, Elton e molti altri profughi come loro sco-

8 Linda era la bellissima amica di scuola di Klea che, a causa di un incidente all'età di dieci anni, aveva perso l'uso delle gambe, per cui si trova sulla sedia a rotelle. Lei aveva un ricchissimo mondo interiore. Le amiche ricordavano benissimo il tema libero che aveva svolto in letteratura al secondo anno del liceo, in cui aveva scritto che sognava di essere una ballerina di danza moderna e che in età avanzata, quando non avrebbe potuto più danzare, avrebbe scritto musica per balletti moderni, dove il corpo sarebbe stato libero di imitare la vita quotidiana "senza irrigidirsi nei movimenti ridicoli, in preda ai crampi, della danza classica”. La sua vita è rimasta preda della mancanza di libertà in tutti i sensi, per cui lei si ribella e, un bel giorno, si impicca. 
prono subito che ci sono dei limiti posti alla loro libertà di movimento, alla possibilità di ricominciare una vita nuova, diversamente da quanto sognato. Si ha a volte la percezione di vivere nella stessa situazione lasciata alle spalle a Tirana.

E violazione di libertà la curiosità degli occidentali durante il soggiorno in Danimarca, anche se di natura diversa da quella degli albanesi, per cui Klea e la sua collega Alba, non ancora profughe, si sentono inferiori in quanto provenienti da un paese arretrato e sconosciuto a tutti.

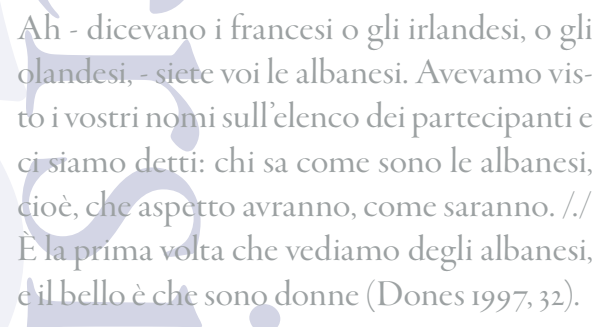

Ma il vero contatto di Klea con l'Occidente è la fuga in Svizzera, dove affronta la vera realtà, dove capisce che nessuno viene accolto a braccia aperte, ricordandole che lei in Svizzera "era una straniera”. I primi tre mesi non le era stato concesso il diritto di lavorare. Ironia della sorte, prima doveva essere vaccinata. Poi doveva passare alla Polizia degli stranieri, che l'avrebbe munita di un permesso di soggiorno provvisorio e di un permesso di lavoro. Dopo mesi di tentativi inutili in cerca di lavoro, avrebbe potuto esercitare la sua professione di insegnante di inglese o qualcosa del genere, seppe che la sua laurea universitaria non era riconosciuta e che avrebbe dovuto sostenere altri esami in una scuola dell'Occidente, a Zurigo o a Lugano. I controlli rigidi della polizia svizzera, che le ricordano quelli albanesi, e l'isolamento, poiché non esiste ancora una diaspora albanese, per cui il suo isolamento è anche linguistico, aggravano i sensi di colpa per aver lasciato la terra nativa e il figlio, tanto che la vita le pare una nuova prigionia in una terra che le dava "solo strade pulite e relazioni ipocrite", (Dones 1997, 227) a cui non poteva essere riconoscente solo perché le aveva dato una dimora e nessun lavoro, dove si sentiva un mostro. È così precorritrice della delusione con la quale si scon- trarono migliaia di profughi albanesi che guardavano all'Occidente come alla terra promessa. Lei capiva benissimo la negazione della giovane donna col figlioletto tra le braccia quando gridava "è deutsch, è nato pochi giorni fa all'ambasciata tedesca, sarà deutsch, non è albanese, maledetta Albania!", come capiva benissimo la speranza dell' illusione che per la donna era là, in Germania, un breve sogno in cui la "Germania li aspettasse a braccia aperte per regalare loro una vita da favola" (Dones 1997, 262).

Klea si sente non di meno oggetto dello sguardo in Svizzera ${ }^{9}$, diversamente da Elton che, nel paese dove dovrebbe avere la massima serenità, rivive stati di ansia provocati dal trauma dell'infanzia, ma non da eventi realmente accaduti $^{\text {10 }}$.

La curiosità va di pari passo con il pregiudizio, "Voi dell'Est avete un piccolo problema con la disciplina al lavoro", dice il padrone del centro commerciale dove Klea riesce a sistemarsi. Saranno episodi frequenti negli anni seguenti e non solo in Svizzera ma ovunque ci fossero i profughi. I padroni della pizzeria non avevano mai mandato giù il fatto che Elton rappresentasse le due categorie di gente da loro più odiate, gli stranieri e gli studenti.

L'emigrante vive nel regno del verbo 'dovere'. Elton, studente universitario in Italia, si mantiene durante gli studi lavorando come in-

9 Nella solitudine in cui è caduta, Klea viene disturbata dai commenti idioti di qualche signora ben vestita e con un trucco orribile, tipo "ah, buonasera signora. Uh, quasi non l'avevo riconosciuta, è la fidanzata del figlio dell'avvocato Montalban. Auguri! La Signora Montalban, la moglie dell'avvocato, mi ha parlato bene di lei, non mi ricordo da dove viene? Ho dimenticato come me l'ha detto." E continuava così tutte le volte che la incontrava a passeggio con il cane: "Quale lingua si parla lì? Non siete cattolici, credo".

Io Elton è diventato claustrofobico a causa di quello che aveva vissuto nel passato; la prigione, l'allontanamento da casa dopo l'arresto del padre, la famiglia spiata dalla Polizia segreta. Da adulto ha paura dei luoghi chiusi, è turbato dai passi lontani di qualcuno, pensando sempre che la sua presenza in un posto sia illecita.

"Quando mi trovo in un ambiente che non conosco non perdo mai di vista l'uscita. Mi viene spontaneo. La forza dell'ignoto ti costringe a metterti di fronte alle più impensate possibilità. Anche una sede qualsiasi può trasformarsi in un non so cosa... quel luogo suscitava paura a me e pena per loro" (Kubati 2004, I27).

I I Del maltrattamento dei giovani albanesi in pizzeria da parte del proprietario Nardon e del suo connazionale Saverio, si legga in Vae non torna, Pp. $145^{-1} 48$. 
terprete e traduttore per il tribunale, facendo il traduttore di intercettazioni, con il lavoro in pizzeria, così come tanti altri come lui, studenti e no, come Leo che non ce la fa più perché:

Quello stronzo di Nardino mi vuole in piz-
zeria fino a tardi, invece a mezzanotte dovrei
prendere il furgone per distribuire i giornali.
/.../ Pagano poco, e la casa come la pago, e le
bollette? /.../ E la bambina quanto mi costa?
Il cibo, i vestitini, le medicine... /.../ e la bol-
letta del telefono, e il bollo della macchina?
No, no, me ne devo andare (Kubati 2004,
28).

Klea pure volava coi pensieri nella terra che odiava e amava allo stesso tempo, nel suo modesto ufficio, nella sua televisione, diversamente dai commenti dei colleghi giornalisti di Ives, secondo cui per lei era "meglio vivere qui che sotto la dittatura” (Dones 1997, 248). Le porte dell'Occidente erano socchiuse. Tutto quello che riuscivano a sapere su di noi dell'Est era solo questo, un pezzo di pane, dimenticando che la vita richiede l'anima per viverla. E, all'anima si erano posti dei confini entro cui venivano inchiodati tutti i fuggiaschi dell'Est.

Gli emigranti devono riuscire nel paese in cui sono venuti, per forza, per non tornare indietro falliti. Va e non torna, il titolo del romanzo di Kubati, si riferisce al folclore balcanico, un motivo ricorrente alla base di tanti racconti. L'eroe della favola (l'emigrazione nel caso nostro) si trova sempre all'incrocio di tre direzioni: va e torna facilmente, va e torna con difficoltà, va e non torna. Le prime due sono favole del fallimento. La terza è simbolo della vita che trionfa:

\footnotetext{
Gli eroi sono sempre giovani. /.../ in realtà non hanno scelta. Tutti s'incamminano in coscienti, per impulso, verso la terza via, verso il futuro che si apre all'inedito, verso un futuro diverso, forse senza prendere neanche sul serio l'ammonimento che non sarebbero più tornati. Le tre vie in realtà coesistono. La narrazione però non può non occuparsi del la vita che passa obbligatoriamente per la terza via (Kubati 2004, 186-7).
}

Usando un motivo fiabesco Kubati ha fatto, in modo metaforico, il bilancio degli albanesi in Italia. Una è la categoria di chi si è lasciato abbandonare ai fenomeni sociali e morali negativi, per loro Elton fa l'interprete durante i processi giudiziari o traduce le telefonate intercettate. Dashuri e huaj, il titolo in lingua originale del romanzo di Dones, Amore straniero, secondo Mauceri sottolinea la relazione sentimentale tra i due personaggi principali, la donna albanese e il giornalista occidentale. Oserei interpretare questo titolo come una metafora dell'amore pazzo degli albanesi verso l'Occidente, tanto da far diventare loro amanti furiosi e non ricambiati nel loro sentimento, cioè rifiutati. L'altra categoria rappresenta chi, come Elton, si è integrato con difficoltà ma con dignità nella società italiana. Il fallimento amoroso non fece arretrare Elton, perché aveva capito che i suoi "bisogni avevano inventato o scoperto Elena”, la ragazza italiana conosciuta tra le aule dell'università, un mezzo per sentirsi realmente integrati nel paese di accoglienza.

"La città alle nostre spalle diventa sempre più piccola, ma davanti a noi non si vede niente" è la frase che chiude il romanzo di Kubati, come forte messaggio della condizione caotica dell'esule, per cui l'Occidente è ancora lontano e che ha fatto solo un salto nel buio. Per citare Dones "Partiamo. Viviamo. Con coraggio. E senza bagagli."

In conclusione, nonostante i romanzi siano autobiografici, con sullo sfondo il contesto della dittatura, raccontano la vita di un' intera generazione di giovani e adolescenti che hanno lasciato l'Albania in cerca di una nuova patria per poter realizzare i sogni.

Tra le righe dei due romanzi emerge la figura dell'emigrante con la sindrome delle frontiere, non solo quelle convenzionali, ma anche quelle invisibili, riscontrabili nella quotidianità. Si lascia scorrere sotto gli occhi la surreale volontà di dare un senso all'abbandono della terra natia, che nei due specifici casi è la fuga da circostanze diverse. 
La sindrome delle frontiere si sviluppa poi nella nevrosi del successo, un successo che conferisce il diritto di restare nella nuova terra. Il doppio punto di vista, di chi è in Albania e di chi, esule, se ne allontana, mette in evidenza, senza fare sconti, la condizione dell'Albania sotto il regime comunista.

Si conclude con la triste riflessione sugli emigranti, condannati ad amare e odiare contemporaneamente la propria terra. La narrazione transita tra l'Albania e l'Italia, per cui il passato ed il presente sono sempre compresenti, la continua alternanza spaziale e temporale, testimonia la condizione dislocata dell'emigrante, creando una discordia tra le due realtà diametralmente diverse.

\section{Povzetek}

Albanski pisatelji nove generacije, ki so se izselili v Italijo, širijo zemljevid transnacionalne literature v Italijo od devetdesetih let dalje. Njihova dela pripovedujejo o resničnih zgodbah ali takih, ki so del kolektivnega spomina, in segajo v čas režima in propada diktature, a tudi v leta izseljenstva in imajo, poleg svoje razvedrilne funkcije, tudi raziskovalno vrednost zaradi njihove psihološke vsebine.

V prispevku obravnavamo dva romana Va e non torna (Pojdi in se ne vrni) Ronija Kubadija in Senza bagagli (Brez kovckkov) Elvire Dones. Oba sta prvenca z izrazito avtobiografsko vsebino, ki obravnavata obdobje diktature in se zaključita z izselitvijo in pristankom na drugi strani morja.

Kubati oriše svojo zgodbo od otroških spominov vse do časa diktature, očetove aretacije in internacije, najstništva in gledanja prepovedanih televizijskih programov, na skrivaj organiziranih plesov, prisiljenega študija, ter se zaključi z opisom študentskega življenja v Italiji ob službi, študiju in napornem preživetju s preskakovanjem med preteklostjo in sedanjostjo ter osebne usode z javnim življenjem v Albaniji, med katerimi izstopa predvsem njegov beg iz domovine.

Življenje protagonistke romana Elvire Dones se zrcali v biografiji avtorice, televizijske delavke, ki opisuje dvojno zgodbo: zgodbo Albanije med osemdesetimi in devetdesetimi leti, v obdobju prehoda iz zatirajočega režima v navidezno demokracijo, ter ljubezensko zgodbo, eno od tistih, ki spremenijo tok življenja. V njeni zgodbi se zrcalijo zgodbe mnogih mož in žena, ki so prečkali Jadransko morje v upanju na boljšo bodočnost, polni zaupanja, ki pa so morali obenem prestati težave izseljenstva, izkoreninjenosti, zavrnitve na domači zemlji, prezrti s strani tistih, s katerimi so do takrat delili svoja življenja.

Oba romana lahko pripovedujemo in obnovimo na treh različnih nivojih, ki se na prvi pogled zdijo enostavni, a v ozadju skrivajo čudoviti svet, ki je razdeljen tudi v realnosti bralca: časovni nivo, ki se razvija v treh obdobjih, tistem v Albaniji, izselitev, proces integracije v novo družbo; prostorski nivo, domovina v odnosu do diktature, simbol kolektivnega zapora in zavedanja, gradnja mobilnosti v tujini, oziroma prazen prostor potovanja proti novemu domu, in končno kraj prihoda; in še tisti psihološki, ki se izkaže iz perspektive, izkušnje in seveda dejanj protagonistov, Eltona in Klee Borova.

Med vrsticami obeh romanov izstopa figura izseljenca, ki trpi za sindromom meje, ne samo tiste konvencionalne, a tudi tiste nevidne iz vsakodnevnega življenja. Pred našimi očmi drsi nadnaravna želja, da bi osmislil umik iz domovine, ki je v obeh specifičnih primerih beg iz različnih okoliščin.

Sindrom meje se nato razvije v iskanje uspeha, ki pa podeljuje pravico, da priseljenec ostane v novi državi. V psihi izseljenca so velike psihološke dileme. Gre za zapleteno osebnost, polno negotovosti, zato se s težavo prepusti pripovedovanju. Počuti se izpostavljenega indiferenci, negotovega, izkoriščanega. Zato da razumemo migranta, je potrebno, da pogledamo v njegovo preteklo zgodbo, tisto pred emigracijo, kar se je zgodilo pred odhodom in oblika dnevnika je za to zelo primerna. Dvojni zorni kot, tistega, ki je odšel, in tistega, ki je ostal, izpostavi stanje Albanije v času diktature, ne da bi pri tem povzročil napetosti.

Članek se zaključizžalostnim razmišljanjem o priseljencih, ki so obsojeni, da ljubijo in istočasno sovražijo svojo zemljo. Spreminjajoče pripovedovanje med Albanijo in Italijo, zaradi katere sta preteklost in sedanjost, so-prisotna, ter neprestana prostorska in časovna alternacija pričata o dislociranem stanju migranta in o nesoglasju med dvema svetovoma, ki sta si diametralno nasprotna. »Mesto za našim hrbtom se manjša, pred nami pa ne vidimo ničesar « je stavek, ki zaključuje Kubatijev roman in simbolizira kaotično sporočilnost migranta, za katerega je Zahod še daleč in nosi občutek, da se je vrgel 
v črno luknjo. Če citiramo Dones: »Gremo. Živimo. Hrabro. In brez kovčkov.«

\section{Summary}

New generation Albanian writers, emigrating to Italy, broaden the geography of transnational literature of today's traditions in Italy, from the 1990s. Their works tell stories that had been lived personally or belonging to collective memory, from the time of the Stalinist regime and the fall of the dictatorship, but also stories during the years of emigration, which, in addition to reading for entertainment, are also worthy texts for the study of psychological nature.

In this short speech we deal with two following novels: Goes and does not come back (Kubati,R. 2004) by Ron Kubati and Without luggage (Dones, E., 2015) by Elvira Dones, both novels being the first two novels for each author, disposing of a strong autobiographical note, referring to the dictatorship period and ending with abandonment, on-the-spot discovery on the other side of the sea.

Kubati shows his own life, memories of childhood during the dictatorship period, the arrest and imprisonment of his father at his teens and the prohibition of watching $\mathrm{TV}$, dancing parties organized in secret, the official determination of pursuing studies, and the narration ends with his student life in Italy among work, studies and efforts of survival, interspersing in a back and forth between the present and the past personal and public events in Albania, the last of which is the flight from the country.

Elvira Dones, whose biography closely resembles that of the protagonist of her novel, a television operator, gives us a double story; the history of Albania between the eighties and nineties, in the period of transition from the oppressive regime to that of an apparent democracy, intertwined with a love story, of those that change life. Through her story the author shapes that of many men and women who have crossed the Adriatic confident in a better future, full of hope and who have faced thousand difficulties of being refugees, uprooted, repudiated at home, despised by people with which for years have shared their lives.

Elvira Dones, whose biography closely resembles that of the protagonist of her novel, a television operator, tells us a double story; the history of Albania between the 80 and 90 , in the period of transition from the oppressive regime to that of an apparent democracy, intertwined with a love story, of those who change their lives. In its history, it shaped that of many men and women who crossed the Adriatic confident in a better future, full of hope and who faced the thousand difficulties of being refugees, uprooted, repudiated at home, despised by people with whom they have shared their lives for years.

Both novels can be narrated and summarized in three cardinal plans which at first sight seem quite simple, but which hide in their background a fascinating world in layers even in the reality surrounding the reader: the temporal, in turn divided into three parts, the stay period in Albania under the communist dictatorship, the exodus, the process of integration into the new acquired reality; the spatial one, the fatherland in relation to the dictatorship, symbol of the collective prison and of awareness, the construction of mobility abroad, namely the empty space of the journey to another home, finally the country of arrival; the psychological one, realized through perspective, experience and certainly the action of the protagonists, Elton and Klea Borova.

Through the lines of the two novels, emerges the figure of the emigrant who presents the syndrome of frontiers, not only the conventional ones, but also those which are invisible and present in everyday life. There remains to us the surreal desire to give meaning to the fatherland abandonment, which in the two specific cases is the escape from different circumstances.

The frontiers syndrome then evolves into the neurosis of success, which confers the right to remain in the new land. Many things happen in the emigrant's psyche. He is a complicated being, he surrounds himself with insecurity, so he does not immediately give in to the narration. He feels exposed to rejection, indifference, unprotected, exploited. To understand an emigrant, his first story must be told, that of the "anti-emigration", the background of the exodus and the diary is a form of it. The double point of view, of those who are in Albania and those who are in exile, undoubtfully highlights the condition of Albania under the communist regime.

It ends with the sad reflection on immigrants, condemned to love and hate their homeland at the same time. The narration moved between Albania and Italy, whereby the past and the present are always present, the 
spatial and temporal continuous alternation, testifies to the displaced condition of the emigrant, creating a discord between the two diametrically different realities. "The city behind us becomes smaller and smaller, but nothing can be seen before us" is the phrase that closes Kubati's novel, as a strong message of the chaotic condition of the exile, so that the West is still far away and has just taken a leap in the dark. To quote Dones "Let's go. We live. With courage. And without luggage."

\section{Riferimenti bibliografici}

Dones, E. 2012. Elvira Dones official website italiano. Http://www.elviradones.com.

Dones, E. 1998. Senza bagagli. Nardò: BESA Editrice.

Dones, E. 2015. Dashuri e Huaj. Tiranë: Botime Pegi.

Federici, A. 2011. “Autopsia dell'animo: la migrazione nei romanzi di Elvira Dones." In Scritture italiane della migrazione, a cura di Ornela Vorpsi e Anilda Ibrahimi, I-2I. Toulose: Line@editoriale.

Gnisci, A. 2003. Creolizzare l'Europa. Letteratura e migrazione. Roma: Meltemi.

Kapllani, M.G. 2016. Ditar i shkurtër kufijsh. Tirane: Nereida.

Kubati, R. 2004. Va e non torna. Nardò: BESA Editrice.

Mauceri, C.M. 2009. “Oltre il muro: dramma personale e nostalgia conflittuale in Dashuri e huaj di Elvira Dones." In La storia nella scrittura diasporica, a cura di Franca Sinopoli, 85-107. Roma: Bulzoni.

Mauceri, C.M.2or3. "Variazioni sul tema dello sguardo nei romanzi d'esordio di Dones e Kubati." In Il confine liquido. Rapporti letterari e interculturali fra Italia e Albania, a cura di E. Bond, D. Comberiati. 185-200. Nardò: BESA Editrice. 